\title{
En busca de la soberanía alimentaria: dos casos de cooperativas de comercio agroecológico en Quito
}

\author{
Susana Anda Basabe* \\ Miguel Durango** \\ Milena Espinosa Manrique ${ }^{\star \star \star}$
}

RESUMEN

EN EL PRESENTE ARTículo SE EXPONEN LOS PRINCIPAles disCURSOS Y PRÁCTICAS de dOS COOPERATIVAS DE

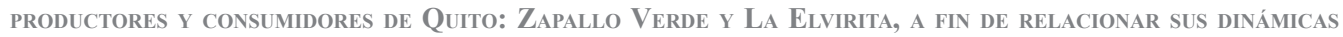
CON LAS PRINCIPALES POLÍticas ALIMENTARIAS de ECUAdOR. LAS ALTERNATIVAS PRESENTAdas APARECEN COMO UN

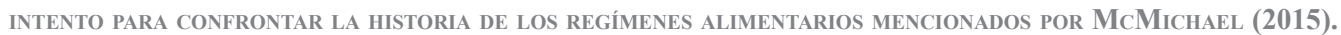
A nUESTRO ENTENDER, ESTAS INICIATIVAS PROYECTAN LA FORMA EN QUE LA AGENCIA DE LOS ACTORES INFLUYE EN LA CONFORMACIÓN DE REDES Y POLÍTICAS, PERMITIENDO UN ACERCAMIENTO HACIA LA CONSOLIDACIÓN DE UN RÉGIMEN DE SOBERANÍA ALIMENTARIA COMO MODELO ALTERNATIVO AL DESARROLLO.

Palabras Clave: cooperativas - Soberanía Alimentaria - Agroecología - Comercio solidario.

\section{Abstract}

THIS PAPER PRESENTS THE MAIN DISCOURSES AND PRACTICES OF TWO COOPERATIVES OF PRODUCERS AND CONSUMERS

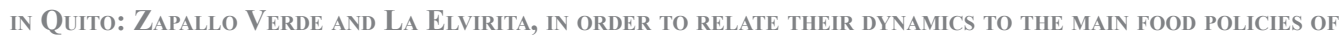
ECUADOR. THESE ALTERNATIVES APPEAR AS AN ATTEMPT TO CONFRONT THE HISTORY OF THE FOOD REGIMES MENTIONED BY MCMichael (2015). In OUR OPINION, THESE INITIATIVES PROJECT THE WAY IN WHICH THE AGENCY OF ACTORS' INFLUENCE IN THE FORMATION OF NETWORKS AND POLICIES, ALLOWING AN APPROACH TOWARDS THE CONSOLIDATION OF A REGIME OF FOOD SOVEREIGNTY AS AN ALTERNATIVE MODEL OF DEVELOPMENT.

KEYWORDS: COOPERATIVES - FOOD SOVEREIGNTY - AGROECOLOGY - FAIR TRADE.

* Investigadora de la Facultad Latinoamericana de Ciencias Sociales - FLACSO Ecuador. Correo electrónico: gsandab@gmail.com.

** Magíster en Estudios Socioambientales de la Facultad Latinoamericana de Ciencias Sociales - FLACSO Ecuador. Correo electrónico: migueldurangoc@gmail.com.

*** Magíster en Ciencias Sociales con mención en Gobernanza Energética de la Facultad Latinoamericana de Ciencias Sociales - FLACSO Ecuador. Correo electrónico: milena_esp@yahoo.fr. 


\section{Introducción}

E 1 actual modelo de desarrollo, asentado en términos de una búsqueda de un crecimiento económico infinito, ha producido, de manera global, desequilibrio y deterioro en la calidad de vida humana y del medio ambiente. Así lo ha descrito O'Connor (2001) en su teoría de la segunda contradicción del capitalismo. Haciendo referencia a la relación entre sociedad, naturaleza y desarrollo, indica que la externalización de los costos sociales y ecológicos derivados de la producción, característicos de un modelo que se orienta hacia la eficiencia, han provocado varias crisis económicas y ecológicas. Dicha problemática es especialmente reveladora en los marcos de disfunción sociopolítica y económica de los años setenta, noventa y en el primer decenio del 2000, época donde se desarrolló una crisis mundial alimentaria que dejó en el 2008, a más de 1020 millones de personas en situaciones alarmantes de hambruna y pobreza (Rosero et al., 2011:14-16).

Lejos de su tratamiento como factores de carácter estructural relacionados con la desigualdad en la redistribución de la riqueza -en el ámbito nacional, regional y mundial-o con la lógica de acumulación del sistema económico mercantil, los problemas del hambre y la pobreza han sido manejados tradicionalmente a través de la cooperación económica y la transferencia de tecnología de los países desarrollados hacia los menos desarrollados (Bretón, 2009). Estas fórmulas, que incluso hoy en día siguen vigentes, apuntan hacia el incremento de la productividad y la reducción de barreras comerciales, pero también hacia condiciones sociales y ecológicas desfavorables, especialmente para los denominados países en vías de desarrollo.

De este modo, a pesar de las grandes inversiones en ayudas, proyectos de desarrollo y transferencia de tecnología, no se han logrado reducir los problemas de hambre, desigualdad y degradación ecológica. Por el contrario, a la vez que los consumidores son afectados por los elevados precios y el difícil acceso a los alimentos, los agricultores campesinos y sus familias son expulsados del mercado como resultado de la caída de los precios de los alimentos, el reducido valor de sus cultivos y los altos costos de los insumos agrícolas. Como si esto fuera poco, Latinoamérica ha priorizado la producción para la agroexportación y los agrocombustibles en lugar del autoabastecimiento, factores que contribuyen directamente a una crisis que traspasa el ámbito rural afectando tanto a productores campesinos como a consumidores (Rosset, 2009; Altieri y Nicholls, 2010).

A nivel microsocial, las dos alternativas que se presentan en este artículo aparecen como un intento de confrontar la historia de los regímenes alimentarios mencionados por McMichael (2015). En este sentido, se analizan los discursos y prácticas presentes en dos cooperativas de productores y consumidores de Quito: Zapallo Verde y La Elvirita, en relación con las principales políticas públicas alimentarias impulsadas en Ecuador. A través de un estudio etnográfico desde el actor, se estudian estas dos iniciativas agroecológicas debido a la trayectoria que han tejido en conexión con prácticas de comercio solidario. Estas iniciativas proyectan la forma en que la agencia de los actores influye en la conformación de redes y políticas, permitiendo un acercamiento hacia la consolidación de un régimen de soberanía alimentaria como modelo alternativo al desarrollo.

\section{Viejas formas, nuevas formas agroalimentarias ¿una oportunidad para el desarrollo?}

Después de la Segunda Guerra Mundial, en el marco de hegemonía de EE. UU., la gran mayoría de organismos de desarrollo implementaron tanto programas de ayuda alimentaria internacional como una serie de políticas económicas que favorecieron la expansión de tecnologías agrícolas. Estos elementos proporcionaron las condiciones suficientes para construir un sistema donde dominaría la producción intensiva de alimentos (Arce y Marsden, 
1993: 293), fenómeno que correspondió a la reestructuración del orden económico mundial bajo nuevos modelos de producción y consumo de alimentos a escala global, lo que McMichael (2015) ha señalado como el segundo régimen alimentario ${ }^{1}$. En este sentido, se impulsó la agroindustrialización, facilitando la estructuración de un sistema de acumulación intensivocapitalista bajo la figura de especialización de mercancías. En efecto, particularmente en Latinoamérica, los agronegocios generaron vínculos transnacionales en los países del Sur, los cuales fueron divididos según su especialización agrícola y determinados por las cadenas globales de mercancías. Esto no solo dio paso a una nueva estructura internacional del trabajo agrícola y a la expansión de las relaciones de mercado, sino también fue la vía por la cual se generó un régimen privado de comercio global (McMichael, 2015).

En la década de 1960, el incremento de la productividad y la introducción de semillas mejoradas y fertilizantes en varios países del mundo dieron paso a la denominada revolución verde, un supuesto logro que se constituyó en ese momento como un proyecto en el que los países subdesarrollados participarían en los mercados globales para solucionar sus problemas de desarrollo. Desafortunadamente, los resultados no fueron los esperados. Particularmente en Latinoamérica, la creencia incondicional en las oportunidades otorgadas por el mercado global animaron la implementación de reformas en las estructuras productivas, que al ser orientadas hacia la intensificación de la producción agropecuaria, implicaron la pérdida de diversificación de productos alimentarios, deterioro ambiental, concentración del sistema alimentario en pocas empresas transnacionales y transformación de la lógica de autoconsumo de los campesinos, vulnerando a la vez su control y acceso sobre las condiciones de producción (Bretón, 2009). En términos ecológicos, durante este proceso se evidenció una remarcable pérdida de flora y fauna, pues a inicios de la década de 1970, el planeta perdió casi un tercio de su vida silvestre (WWF, 2008).

Desde la década de 1950 hasta la actualidad, la ciencia y la tecnología, bajo un enfoque económico-productivista, han sido componentes que se han considerado más que fundamentales para el incremento de la producción. En efecto, al mismo tiempo que los sectores campesinosagrícolas han reducido su capacidad para autoabastecerse de alimentos cultivados por ellos mismos y, por el contrario, han aumentado su dependencia del conjunto de multinacionales que controlan una parte significativa del proceso alimentario; las grandes empresas transnacionales han logrado no solo controlar el sector de producción primaria, sino también empaquetar, ampliar y reorientar el tiempo de producción, así como manipular la calidad y el contenido de los productos alimentarios generando un valor añadido y una reconfiguración del tiempo necesario para realizar dicha tarea (Arce y Marsden, 1993).

Sin embargo, según McMichael (2015), desde la década de 1980 hasta el presente han existido divergencias. El autor afirma que el mundo experimenta un nuevo régimen, un tercer régimen alimentario, cuyo orden se estructura en el poder corporativo financiero, caracterizado por la hegemonía de las grandes empresas agroalimentarias transnacionales, el capital financiero, la internacionalización de la agricultura, entre otros aspectos. Este nuevo régimen es de orden neoliberal, pues ya no son las corporaciones privadas las principales protagonistas sino los gobiernos, quienes sirven a los mercados y a las corporaciones transnacionales ${ }^{2}$. De hecho, este nuevo orden se caracteriza por la combinación del monopolio privado y la promoción de subsidios gubernamentales de los países del Norte, lo cual ha

1 McMichael (2015) identifica tres regímenes alimentarios: régimen centrado en Inglaterra (1870-1930), régimen centrado en EE. UU. (1950-1970) y régimen alimentario centrado en el poder corporativo financiero (1980-actualidad). Cada régimen alimentario se define como un orden económico mundial mediado por normas y relaciones políticas y económicas, correspondientes a las coyunturas geopolíticas dominantes, que estructuran la producción y el consumo de alimentos a escala global.

2 Según McMichael (2015), el tercer régimen alimentario surgió en el contexto de crisis de deuda y ajustes estructurales neoliberales en el Sur, y fue posible por la influencia política de organizaciones internacionales, principalmente la Organización Mundial del Comercio (OMC) y el Fondo Monetario Internacional (FMI), quienes promocionaron políticas a favor del libre comercio, las potencias mundiales y las grandes empresas transnacionales. 
representado una gran desventaja para los agricultores latinoamericanos hasta el punto de sufrir desplazamiento y despojo, pues es imposible competir con los grandes monopolios o los mercados subsidiados.

Aparte de las problemáticas sociopolíticas y económicas señaladas, el modelo agroindustrial presenta grandes riesgos ecológicos: es altamente contaminante. Pero sobretodo, ha surgido una nueva problemática en relación al estancamiento de la producción agrícola por medio de insumos químicos, puesto que ya no presenta los mismos índices de productividad que a inicios de la década de los setenta. En busca de una respuesta económicamente eficiente, en 1992 se impulsó el desarrollo de plantaciones transgénicas, donde China consta como uno de los primeros países en implementar dichos cultivos. Desde finales de la década de los noventa, dos fueron los países de América Latina, Argentina y México, en adoptar estos cultivos (Larach, 2001). Hoy en día, el debate no solo se ha centrado en el incremento productivo agrícola sino también sobre el control transnacional de las semillas, pues estas plantaciones han aumentado en diferentes países de Sur.

Precisamente, frente a estos escenarios contradictorios han surgido varias organizaciones a nivel mundial (como La Vía Campesina) para defender la alimentación como un derecho humano y no como una mercancía. Particularmente en Ecuador se han generado una serie de iniciativas políticas y organizativas, tanto de parte de los productores como de los consumidores, quienes en resistencia al modelo agroindustrial se han preocupado por cultivar alimentos a baja escala, sostenibles, sanos, nutritivos y culturalmente apropiados en pro de la soberanía alimentaria. Dichas alternativas proponen formas alternas de producción, comercialización y consumo, que buscan enfrentar la pobreza y la desigualdad de condiciones agroproductivas en el país. En los siguientes acápites se señalan que son varios los autores que han apoyado este tipo de iniciativas, enfatizando en la importancia de la participación de movimientos y luchas sociales para la transformación de políticas públicas a favor de un régimen de soberanía alimentaria.

\section{Soberanía alimentaria, agroecología y políticas públicas de alimentación en Ecuador}

En un escenario en el que el actual modelo de desarrollo económico ha causado problemas crecientes a nivel global, la propuesta de soberanía alimentaria y dentro de ella la agroecología se han presentado como alternativas para enfrentar las problemáticas que ocasiona el sistema agroalimentario industrial. La noción de soberanía alimentaria es una noción política y práctica. El concepto surgió por primera vez en 1996, como una iniciativa de La Vía Campesina y otras organizaciones sociales. En Ecuador, este concepto ha sido adoptado dentro de la actual Constitución como un eje transversal de diferentes programas del Estado, plasmados en el Plan Nacional de Desarrollo y en la Ley Orgánica del Régimen de Soberanía Alimentaria del 2009.

Algunos autores como O'Connor (2001) y McMichael (2015) han señalado que para superar el modelo vigente de eficiencia productiva-económica, los agentes principales de cambio son los movimientos y las luchas sociales. Dentro de ello, el Estado y la familia sostienen especial relevancia, puesto que despliegan relaciones sociales de reproducción de las condiciones de producción. De este modo, el concepto de soberanía alimentaria es de particular importancia, ya que sostiene como base fundamental el abastecimiento de alimentos a nivel familiar, luego local y posteriormente nacional. Este principio responde no solo a la importancia de la capacidad de decisión de los actores sociales sobre la producción, distribución y consumo, sino también al control de todo el circuito agroalimentario a través de modos sustentables y culturalmente apropiados (Montagut, 2011). Para que dichas prácticas se lleven a cabo, el Estado tiene un rol fundamental, el de garantizar no solo el acceso a alimentos, sino el control de estos, en tanto que productores como consumidores se constituyan como actores autónomos e independientes de las dinámicas del mercado global (Red de Guardianes de Semillas, 2007). 
En este sentido, las prácticas sustentables y agroecológicas ejercidas por las familias son elementos más que fundamentales para la construcción de un régimen de soberanía alimentaria, pues desde esta perspectiva proponen una articulación entre los agricultores y los consumidores, promoviendo el control del sistema agroalimentario y la dinamización del sector rural (Altieri y Toledo, 2011; Andrade y Flores, 2008; Rosset, 2004). Desde la praxis social y en resistencia a los problemas de las crisis globales, varias personas y organizaciones han empezado paulatinamente a tomar conciencia de las limitaciones del modelo de agricultura industrial en Latinoamérica. Con o sin el apoyo del Estado, han fomentado la transición hacia un modelo que no sea tan dependiente del petróleo. De este modo, han encontrado espacios donde se incentiva la producción de pequeños agricultores, a la vez que han exigido a los organismos estatales el acceso a los recursos necesarios como tierra, agua, semillas y créditos; el impulso de tecnologías agroecológicas; la promoción de precios justos; y el acceso a los mercados locales.

Particularmente en Ecuador, cada vez existen más familias que se abastecen de alimentos sostenibles mediante sistemas de distribución solidaria, acudiendo a estrategias como las de las canastas comunitarias o las ferias agroecológicas (Red de Guardianes de Semillas, 2007). La reproducción de estas iniciativas se fundamenta en las bases de la agroecología, una opción que se torna viable a medida que sostiene herramientas tanto científicas como metodológicas para la producción de alimentos desde la agricultura campesina y familiar. Esta técnica se constituye como complemento de la propuesta de soberanía alimentaria, en tanto que las prácticas agroecológicas se realizan reconociendo las limitaciones ambientales, energéticas y financieras, y facilitando la conservación de la biodiversidad (Altieri y Nicholls, 2010: 64). En este sentido, las iniciativas de producción y comercio agroecológico se identifican como alternativas social y ambientalmente sostenibles, pues respetan la capacidad de carga de los ecosistemas sin acudir a la utilización de agroquímicos, por lo que dejan a un lado la dependencia de los combustibles fósiles e insumos costosos. A la vez que promueven una agricultura diversificada, resiliente al cambio climático y eficiente energéticamente, contribuyen al fomento de la soberanía alimentaria, energética y tecnológica (Perfecto et al., 2009).

\section{Políticas públicas para el impulso de la soberanía alimentaria y agroecología en Ecuador}

En Ecuador se han establecido políticas que apuntan a un nuevo modelo de desarrollo en relación con la soberanía alimentaria. En este sentido, la Constitución del 2008 es de gran interés, pues recoge a través de un nuevo paradigma de desarrollo - el Buen Vivir- la visión de soberanía alimentaria como objetivo estratégico. Particularmente los artículos 281 y 282 reflejan el establecimiento de un régimen constructor de la Soberanía Alimentaria. En ellos se señala que es una estrategia y una obligación que el Estado debe cumplir para garantizar la autosuficiencia en alimentos sanos y culturalmente apropiados de forma permanente a personas, comunidades, pueblos y nacionalidades. Más aún, la Constitución resalta, en el artículo 400, el valor de la agrobiodiversidad como patrimonio cultural para garantizar el abastecimiento de suficientes alimentos sanos y nutritivos en concordancia con las personas y comunidades de diferentes culturas; y en el artículo 401, "se declara al Ecuador libre de cultivos y semillas transgénicas". En este contexto, nos encontramos frente a un impresionante marco legal que protege los derechos de la naturaleza, los saberes ancestrales, la soberanía alimentaria e incluso la agroecología, nos atrevemos incluso a decir que de entrar adecuadamente en la práctica, podría ser un modelo a nivel mundial para el fomento de la soberanía alimentaria en otros países.

A la vez, la Ley Orgánica del Régimen de la Soberanía Alimentaria (LORSA), aprobada en el 2009, establece diversos mecanismos por el cual el Estado debe cumplir con su obligación y objetivo estratégico de soberanía alimentaria. Esta ley reivindica al productor y al consumidor como los ejes centrales de la cadena agroalimentaria, priorizando los mercados nacionales antes que la producción para exportación y la importación de alimentos de otros países (Borja 
Hidalgo, 2009). Dicha ley se basa en el artículo 281, numeral 6 de la Constitución, que dicta "promover la preservación y recuperación de la agrobiodiversidad y de los saberes ancestrales vinculados a ella; así como el uso, la conservación e intercambio libre de semillas". Asimismo, en su preámbulo retoma el objetivo estratégico de la Constitución de velar por el derecho de las poblaciones a la alimentación, de preferencia basada en una producción local, agroecológica y orgánica, y culturalmente apropiada. De modo que la LORSA expone la necesidad de establecer mecanismos para el cambio de modelo productivo de uno convencional a uno agroecológico, evidenciando la importancia de regular y fomentar las prácticas de manejo de la agrobiodiversidad con un enfoque agroecológico.

Los orígenes de este tipo de acciones orientadas a construir políticas públicas en materia de soberanía alimentaria emergieron de la organización de varios sectores. En el marco político nacional, por ejemplo, se conformó una comisión encargada de preparar y presentar las propuestas de ley necesarias para complementar y hacer viable este nuevo régimen, la Conferencia Plurinacional e Intercultural de Soberanía Alimentaria (COPISA). Este organismo del poder ciudadano es una institución autónoma, que ha contado con la participación de organizaciones de la sociedad civil e instituciones del Estado. Algunas propuestas incluyen la Ley Orgánica de Agrobiodiversidad, Semillas y Fomento Agroecológico, la cual pretende "recuperar, conservar y promover la agrobiodiversidad y fomentar la Agroecología para alcanzar la Soberanía Alimentaria y el Sumak Kawsay" (COPISA, 2011); la Ley Orgánica del Régimen de la Soberanía Alimentaria (LORSA, 2009); entre otras leyes como las de tierras, agua, mangles, etc., que están en debate en la Asamblea Nacional. Así, a través de la COPISA se desprendieron diversas estrategias de los movimientos sociales de interés agrario, aportando a estos procesos políticos para que reflejen las diversas realidades de la producción agrícola nacional.

Por su parte, el fomento de la agroecología en el país surge desde las bases de la producción agrícola. Varios proyectos resaltan el esquema de producción agroecológica en Ecuador y son llevados a cabo por diversas organizaciones sociales y políticas campesinas. En este contexto, el Gobierno Autónomo de la Provincia de Pichincha (GADPP) en diversas reuniones de las mesas agrarias redactó la "Ordenanza para Fomentar la Producción de Alimentos Agroecológicos", que está próxima a entrar en vigencia y contempla la regularización de los procesos de comercialización mediante consejos de participación en diversos niveles para acoger la mayor cantidad de productores agropecuarios. Dentro de esos procesos, el mismo GADPP ha desarrollado un proceso de certificación agroecológica al cual los productores deberán someterse para formar parte de los consejos de productores y de los beneficios.

Sin embargo, tanto los lineamientos de la Constitución como las leyes y ordenanzas vinculadas a la soberanía alimentaria y la agroecología pueden no estar contemplando los procesos vigentes de organizaciones y movimientos sociales autónomos, como las experiencias que se presentan más adelante, que han surgido a partir de la necesidad y se han fortalecido por la agencia personal y colectiva para mantener familias y no solamente producciones y mercados viables. De esta manera, la regularización por parte del Estado y los gobiernos locales puede llevar a la ruptura y colapso de las iniciativas en proceso, ya que imponen relaciones con el mismo Estado y con otros mercados que pueden volver económicamente inviables procesos productivos y de comercialización solidaria.

\section{Limitaciones de las políticas públicas de soberanía alimentaria en Ecuador}

En contradicción con este nuevo paradigma, las políticas públicas en la producción agropecuaria se han orientado hacia la producción agroindustrial y la lógica mercantilista, lo que Van der Ploeg (2010: 310) denomina "economías virtuales", respondiendo a la idealización del campesinado por parte de quienes toman las decisiones políticas. Estos actores desde las esferas de poder poseen una noción del campesinado que invisibiliza otros modos de producción 
y reproducción social de las diversas poblaciones de productores agrícolas. En este sentido, la asignación de recursos apuntala una forma de producción modelo (a mediana y gran escala) que responde a exigencias económicas mercantilistas, dejando en segundo plano a otras realidades de la producción agrícola ecuatoriana, fenómeno reconocido por Carrión y Herrera (2012), quienes destacan que:

Las familias campesinas de pequeños productores, en el marco de las políticas de fomento agropecuario, no son reconocidas como agentes que aportan productivamente al país, sino, que deben ser apoyadas por el Estado para mínimamente sobrevivir. No se valora el potencial productivo de la economía familiar campesina [...] (Carrión y Herrera, 2012: 71).

Para lograr regular este escenario, en el contexto de la producción agropecuaria varios movimientos sociales y políticos relacionados aportaron en normar las políticas públicas. La Vía Campesina; la Confederación Nacional de Organizaciones Campesinas, Indígenas y Negras (FENOCIN); la Federación Nacional de Trabajadores Agroindustriales Campesinos e Indígenas Libres del Ecuador (FENACLE); entre otros apoyaron proyectos de ley para instaurar la soberanía alimentaria como un eje normativo imprescindible para complementar los puntos trazados desde varios acuerdos plasmados en la Constitución vigente. A pesar de ello, las dificultades para establecer un régimen de soberanía alimentaria continúan, especialmente al vincular las normativas expresadas en la Constitución con los indicadores socioproductivos.

Esta problemática es especialmente visible en el artículo 281, el cual contiene 14 literales. Entre ellos, llaman especial atención los literales 1-3 y 6 donde se expresa que el Estado debe impulsar la producción agroalimentaria y pesquera -específicamente para las unidades de producción pequeñas y medianas, comunitarias y de economía social y solidaria-, a la vez que tiene la responsabilidad de adoptar una serie de políticas para evitar la dependencia de importaciones de alimentos, debe fortalecer la diversificación e introducción de tecnologías ecológicas para la producción agropecuaria, y promover la preservación de la agrobiodiversidad y los saberes ancestrales. Los lineamientos de la Constitución protegen, al menos en lo normativo, el sector agrícola con miras a fortalecer el desarrollo de un régimen de soberanía alimentaria. Sin embargo, en la última década, en Ecuador se han detectado tres tendencias en el desarrollo agrícola: 1) la creciente industrialización y aumento de las exportaciones agrícolas; 2) la dependencia de insumos urbanos; y 3) el incremento de poder de la demanda urbana a través de supermercados, agroindustrias y exportadoras (Idrovo, 2016).

En cuanto a los lineamientos señalados, es de gran interés señalar que el país ha incrementado considerablemente sus importaciones de fertilizantes y plaguicidas, y ha implementado una mayor cantidad de monocultivos, que representan el 92\% del área nacional dedicada a la agricultura, lo cual impulsa el desarrollo de sistemas que evidentemente difieren de la preservación de la agrobiodiversidad y de la reproducción de los saberes ancestrales. Asimismo, aunque la tendencia de exportación entre el 2009 y el 2014 puede percibirse como ligera, en este periodo igualmente se evidenció un incremento de 30\% (Ibíd.). En otras palabras, este fenómeno, que indica la producción de un sector agrícola orientado a la exportación, también puede reflejar una creciente dependencia de importaciones para alimentar a su población. De hecho, si bien la mayor demanda de alimentos ecuatorianos es satisfecha con la oferta nacional de pequeños y medianos agricultores, en los últimos años ha existido un incremento en la importación de productos $^{3}$. Indiscutiblemente, estos componentes resultan desfavorables tanto para la producción de economías sociales y solidarias como para las pequeñas unidades de producción enfocadas en producir alimentos a baja escala y agroecológicos. En efecto, reflejan la debilidad de políticas orientadas a garantizar una producción agroalimentaria autosuficiente.

3 Según los datos del Banco Central (BCE), en el período 2002-2007, el consumo interno de alimentos importados fue de $12,58 \%$, mientras que en el período 2008-2010, el consumo aumentó a 16,02\% (Novoa, 2013). 
Por otro lado, dentro del mismo artículo 281 de la Constitución, los literales 10 y 11 indican que es responsabilidad del Estado impulsar el desarrollo de organizaciones y redes de productores y consumidores, así como de comercialización y distribución para generar equidad entre los espacios rurales y urbanos; también indica que el Estado debe "generar sistemas justos y solidarios de distribución y comercialización de alimentos. Impedir prácticas monopólicas y cualquier tipo de especulación con productos alimenticios". Sin embargo, existe una significativa dependencia de insumos urbanos en la cual, la creciente dependencia del sector agrícola a las dinámicas del mercado urbano ha animado al incremento de la productividad agrícola con calidades específicas a bajo costo y mayor industrialización y empaque. Más aún, se ha desarrollado una creciente demanda urbana especialmente en los ámbitos de supermercados, agroindustrias y exportadoras (Ibíd.). Estos elementos reflejan una relación desfavorable, particularmente en la protección de productores, consumidores y redes de sistemas justos y solidarios.

Bajo este panorama, es importante mencionar el incremento significativo de supermercados que ha experimentado el país. Si bien en 1999 se registraron 7 supermercados, esta cifra fue aumentando considerablemente hasta llegar a 67 establecimientos en el año 2014 (Ibíd.: 29). Los indicadores señalan un gran reto para desarrollar alternativas al modelo agroindustrial, pues si este patrón persiste, se incrementará aún más la concentración de la oferta agroalimentaria en los supermercados, lo que no solo se traduce en la homogeneización de la producción y consumo de alimentos, sino también en la desigualdad de oportunidades para los pequeños productores frente a los grandes beneficios que adquieren los productores con sistemas tecnificados y plantaciones de monocultivos a gran escala, aspectos que favorecen la oferta en grandes dimensiones, con calidad especializada y precios considerablemente más bajos que los de los pequeños productores. En definitiva, se advierte una debilidad de la Constitución al no otorgar a los productores y consumidores la capacidad de decisión sobre la producción y el consumo.

\section{Cooperativas de comercio solidario}

Como se ha indicado, cada vez se dificulta más el acceso a un sistema alimentario económico y saludable, pues el mercado moderno separa a los productores rurales de los consumidores urbanos, los intermediarios tienen el control de la distribución y los precios de los alimentos, y, finalmente, los afectados son tanto los productores como los consumidores por los altos e injustos precios de productos que carecen de buena calidad (Kirwan, 2008: 26). En este sentido, las problemáticas relacionadas con el actual modelo agroindustrial se evidencian fundamentalmente en un marcado distanciamiento entre productores y consumidores. Este fenómeno corresponde especialmente al caso de los supermercados donde las frutas, las verduras y otros productos alimenticios se venden a largas distancias de donde se produjeron originalmente, no existe ninguna relación entre productores y consumidores, a la vez que se genera un desconocimiento y falta de interés generalizado por parte de los consumidores sobre la producción, su contenido sociocultural y su procedencia. En pocas palabras, los alimentos se transforman en un bien comercial ausente de significado social.

Por otro lado, conforme se ha tomado mayor conciencia sobre las prácticas agrícolas industriales poco responsables, socialmente excluyentes y ecológicamente perjudiciales, han surgido iniciativas organizativas de gran importancia, donde prevalece la preocupación por cultivar alimentos social y ecológicamente sostenibles, y por consumir alimentos sanos y nutritivos. De este modo, las cooperativas son de gran interés por cuanto buscan enfrentar los problemas de pobreza y las transformaciones ecológicas que ha sufrido el planeta en las últimas décadas. En efecto, nacen como una respuesta de resistencia al modelo agroalimentario dominante. 
Las cooperativas se caracterizan por ser asociaciones autónomas de personas que se han organizado de forma voluntaria con la finalidad de satisfacer sus necesidades. Si bien desde inicios de la década de 1980 se empezaron a visibilizar procesos que se aproximaban a la consolidación de cooperativas en Ecuador, es quizás en la última década donde se ha generado un mayor reconocimiento global del valor de este tipo de asociaciones. De hecho, las Naciones Unidas proclamó en el 2012 como el Año Internacional de las Cooperativas, reconociendo su importancia para el desarrollo económico y social, así como para la erradicación de la pobreza (FAO, 2012; ONU, 2011).

Los antecedentes de los actuales procesos cooperativos de alimentos se pueden encontrar desde 1987. En este año, en Riobamba se agruparon 25 familias pertenecientes a una iglesia local y fundaron la primera canasta comunitaria del país. La organización respondió a la necesidad de buscar alimentos de buena calidad y a bajo precio, "mientras ponían en práctica el concepto religioso de multiplicación de los panes" (Garcés y Kirwan, 2009: 10). Sin embargo, después de once años de funcionamiento, la organización se disolvió. Un año después, en el 2000 surge en la misma ciudad una nueva organización de canastas comunitarias y, desde entonces, las iniciativas se han incrementado en diferentes regiones del país (Kirwan, 2008).

$\mathrm{Si}$ bien las primeras canastas comunitarias tuvieron como objetivo fundamental la adquisición de alimentos sanos a precios asequibles, para lo cual compraban la comida en los mercados locales al por mayor con un consecuente ahorro; muchas de las actuales iniciativas no solo buscan alimentos a precios más bajos, sino que tengan relación con la producción agroecológica y con la garantía de reproducción de un régimen de soberanía alimentaria. En este sentido, las canastas se componen de alimentos básicos a los que todas las personas deberían tener acceso, además de ofrecer a los consumidores mayor variedad de frutas, legumbres, hortalizas, etc. y, por tanto, una dieta mucho más nutritiva a la convencional, permitiendo a su vez recuperar variedades locales de cultivos que se han ido perdiendo y recetas tradicionales. Hoy en día, las canastas comunitarias siguen siendo organizaciones urbanas y populares donde participan tanto hombres como mujeres, representando una alternativa a los mercados modernos y convirtiéndose en una opción para las familias de escasos recursos, donde prevalecen las relaciones directas entre productores y consumidores (Kirwan, 2008; Kirwan y Garcés, 2009).

Aparte de esta iniciativa histórica, existen actualmente otras propuestas que se centran específicamente en la adquisición de productos agroecológicos ofrecidos en ferias o mercados alternativos. Alternativas como la Cooperativa Zapallo Verde o La Elvirita establecidos en la ciudad de Quito, responden a otras condiciones, en este caso, los consumidores son personas que no necesariamente carecen de recursos económicos, pero que buscan consumir productos saludables encontrando en las canastas una opción con variedad y calidad, a la vez que apoyan la producción de los pequeños agricultores. En este sentido, podemos plantear que las dinámicas alimentarias de un sector de la población de la capital han cambiado con el tiempo, favoreciendo el consumo de alimentos promocionados como tradicionales, sanos y altamente nutritivos.

En cualquiera de estos casos, las cooperativas desempeñan un papel clave en la satisfacción de estilos de consumo sanos, diversos y con identidad, ofreciendo una nueva oportunidad para distanciarse de las dietas convencionales. Pero también han demostrado una gran capacidad de adaptación a las crisis alimentarias y económicas, impidiendo que muchas familias caigan en la pobreza. Por lo tanto, la importancia de identificar las relaciones entre productor y consumidor radica en centrar la atención en el consumo, la diversificación de alimentos, la producción y los contextos culturales, pues solo de este modo se podrán visibilizar los cambios sociales que impulsan los actores locales, las empresas pequeñas y familiares agrícolas, e incluso las instituciones estatales. 


\section{Iniciativas agroecológicas de comercio solidario: Zapallo Verde y La Elvirita}

Desde el análisis de las perspectivas de los actores propuesto por Long (2007) se explica a la sociedad a partir de su condición de heterogeneidad que permite la manifestación de una serie de formas sociales y culturales. Por lo tanto, se toman en cuenta las formas variadas de los viejos y nuevos modos de producción, circulación y consumo que generan diversos modelos de cambio económico y cultural. De esta manera, los actores y sus intereses reestructuran los procesos políticos y de desarrollo vigentes, permitiendo así que los actores sean partícipes del cambio. Bajo este marco, el enfoque orientado al actor reconoce cómo los modos de vida y los procesos de los actores junto con sus estructuras son transformados y mediados por estos mismos actores a través de la interfaz y agencia, que permiten construir estrategias de vida frente a circunstancias dificultosas. Asimismo, las diversas formas organizativas son el resultado de las interacciones, negociaciones y forcejeos que se dan en una arena entre distintos actores con distintos intereses (Long, 2007: 39-43). A partir de esto, fue indispensable realizar un estudio etnográfico desde el actor para indagar sobre la heterogeneidad de prácticas de producción, consumo y circulación que se presentan como modelos alternativos a los dominantes.

Hace más de una década ambos colectivos, la Cooperativa Zapallo Verde y La Elvirita, formaban parte del mismo grupo de comercialización en el cual se organizaron los productores para acceder a un espacio dentro de la ciudad de Quito. La finalidad era encontrarse y vender directamente sus productos a los consumidores de una manera responsable, solidaria y participativa. Ambas cooperativas abastecen hoy en día a los consumidores de productos agrícolas, productos elaborados según las épocas de cosecha y alimentos producidos bajo técnicas agroecológicas. Para las dos cooperativas, el objetivo fundamental no es crecer en cantidad de consumidores ni en volúmenes de venta, sino que las actividades de producción, distribución y consumo ecológica y socialmente sostenibles se mantengan en el tiempo para fortalecer la soberanía alimentaria, incitando a que estas iniciativas se repliquen con otros colectivos en el resto de barrios.

Debido a diferentes situaciones e intereses, una parte de los fundadores de la Cooperativa Zapallo Verde decidió abrir un nuevo espacio de encuentro en Tumbaco, de similares características, cerca de su localidad de producción y con mejores garantías para la participación en la producción agroecológica (R. Simbaña, entrevista, 2012). Esta ruptura es considerada como una experiencia constructiva, ya que ambas cooperativas mantienen la misma base ideológica al haber conseguido no crecer, sino multiplicar esta iniciativa. Actualmente se consolidan como colectivos que pueden ayudar a la formación y organización de estas actividades para expandirlas inclusive hacia otras regiones.

\section{Zapallo Verde}

La Cooperativa Zapallo Verde $(\mathrm{CZV})$ es una red que busca la producción y consumo responsable mediante la generación y circulación de alimentos cultivados no solo orgánicamente, sino sobre todo con base en los principios de la agroecología (Fundador CZV, entrevista, 2012). Según la cooperativa, los modelos comerciales promovidos por los grandes supermercados tienen como único fin aumentar las ganancias económicas y, por lo tanto, todos los productos -incluidos los productos orgánicos-se producen en un contexto poco sostenible con el medio ambiente y reproducen patrones de sobreexplotación con los agricultores. Por el contrario, Zapallo Verde surge en búsqueda de formas alternativas de vida, en las que se apoya el respeto a los productores agrícolas, el acceso a alimentos que sean cultivados de modo sustentable y la eliminación de los antiguos patrones de crecimiento económico que no permiten una vida humanizada. Asimismo, en términos de salud, le apuesta a la autogestión de la producción y consumo de alimentos sanos, locales y nutritivos (Fundador CZV, entrevista, 2012). 
La Cooperativa Zapallo Verde se estructura en tres partes: 1) los coordinadores; 2) los consumidores; y 3) los productores, que a su vez venden sus propios productos colaborando con el $20 \%$ de sus ganancias para el espacio. Los coordinadores pueden estar compuestos por productores o consumidores y se alternan por distintos periodos, favoreciendo la transparencia y participación de los actores, y son los encargados de organizar la producción y la venta de productos. En cambio, los consumidores son actores que exigen calidad en sus productos y aportan con iniciativas relacionadas a la cooperativa, esta dinámica se complementa en las asambleas que surgen desde la misma cooperativa. Para acceder a la compra de los productos de la cooperativa, los consumidores pueden asistir directamente para comprar productos excedentes o pedir canastos con alimentos seleccionados según la temporada de cosecha a través del pago inicial de quince dólares, aporte simbólico que permite a los consumidores comprometerse con la cooperativa y que se establece como forma de garantía para los productores. Los canastos se piden por internet cada semana y se retiran cada miércoles en el sector de la Floresta, entre 5 y 7 p. m. Los productores que participan en esta red no buscan un beneficio económico monetario directo ni expandirse bajo la lógica de los supermercados, existe algo más allá del simple hecho de comercializar productos para obtener una ganancia. Según uno de los fundadores de la Cooperativa, lo que realmente se busca es construir el Sumak Kawsay (Buen Vivir en kichwa) y la soberanía alimentaria, pero para ello, dentro de la cooperativa se siguen ciertos reglamentos.

Algunos ejemplos son los que brindaron varios de los productores, quienes afirman que los productos que se ofertan entre distintos productores no pueden ser los mismos porque esto incentiva a la competencia. Por ejemplo, no pueden existir dos ofertas del mismo tipo de lechuga, si existen diferentes productores de lechuga, cada una debe tener características particulares, pues cada productor debe ofertar un alimento distinto. Esta norma de la cooperativa es indispensable para evitar conflictos internos y promocionar diversidad de alimentos. Por otro lado, una de las productoras de alimentos elaborados señaló que el proceso

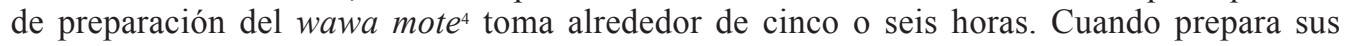
alimentos, debe moler el mote y los chochos, trabajo que requiere un gran esfuerzo de energía humana, dado que los instrumentos utilizados son manuales y no eléctricos, sus productos se venden a baja escala para que no impliquen pérdidas y para que siempre permanezcan frescos. Por otro lado, uno de los panaderos manifestó que utiliza ingredientes sin químicos e instrumentos tradicionales, pero su trabajo no queda en la fabricación de pan, pues no utiliza intermediarios para comercializar su producto, sino que viaja cada miércoles desde Machachi hacia la capital para ganar aproximadamente cinco dólares. Estos casos muestran que la CZV se consolida como un lugar donde nace una alternativa de participación, sin intermediarios, donde los productores son los mismos que comercializan y que su sentido de ganancia no se reduce a fines meramente lucrativos.

En este contexto, una propuesta que contiene este tipo de componentes, como utilización de ingredientes sin químicos e implementos tradicionales, ausencia de intermediarios, comercio entre productores y consumidores, y comercialización de sus propios productos, aunque no impliquen mayores ganancias, es una iniciativa que se orienta más que hacia el nivel económico, hacia la acción política para la transformación social y de las formas de producción dominantes. En este sentido, los productores deben cultivar bajo los lineamientos de la agroecología, no pueden utilizar químicos ni fertilizantes para mejorar su producción ni mucho menos comercializar productos de monocultivos; además, al comercializar los alimentos, deben reducir al máximo el uso de bolsas plásticas. Para los productos elaborados, se pone especial énfasis en el tiempo y esfuerzo que se dedican, reconociendo los costos asociados; también se deben evitar los empaques de plástico en su comercialización. Bajo este panorama, a nivel microsocial, se reduce la dependencia de las multinacionales tanto por parte de los productores como de los consumidores.

4 Tamal elaborado con mote molido, queso crema y pimienta picada, envueltos en una hoja de col. 
Existen cinco objetivos fundamentales de la CZV que se direccionan hacia diversos ámbitos de la producción, el consumo y el comercio, que además se entrecruzan. Todos estos objetivos buscan construir la soberanía alimentaria a través de la creación de una cultura alimentaria sana y con identidad, y mediante una forma de vida basada en alimentos locales y ecológicos (Cooperativa Zapallo Verde, 2008):

1. En primer lugar, se persigue un desarrollo alternativo que sea amigable con el medio ambiente, por lo que se busca producir, comercializar y consumir de la manera más ecológicamente posible. En este contexto, los productores tienen la obligación de cultivar alimentos sin uso de agroquímicos ni pesticidas y tampoco pueden utilizar modelos de producción a gran escala como los monocultivos (Coordinadora CZV, entrevista, 2012). Por otro lado, con respecto a la comercialización de los productos agrícolas, lo idóneo es fomentar la producción local y de comercio con circuitos cortos.

2. Otro objetivo estructural está centrado en las formas de producción y condiciones de los productores. El trasfondo de esta propuesta consiste en romper con los patrones de sobreexplotación e irrespeto que sufren los pequeños productores cuando se ven involucrados en procesos de producción a gran escala. Para ello, se refuerza: a) el pago justo por el trabajo de los productores, b) la existencia de condiciones laborales seguras y saludables, c) la recuperación de los conocimientos tradicionales, d) la recuperación del respeto y la dignidad, y e) la participación de los productores en la cadena de comercio.

3. El tercer objetivo se articula al ámbito del comercio, donde se incentiva el pago justo a los productores y el establecimiento de precios independientes de los constituidos en el mercado convencional. Para esto, es fundamental el apoyo a la relación entre productores y consumidores, eliminando a los intermediarios quienes en el mercado convencional siempre se encuentran presentes e intervienen en las estructuras de producción y comercio de los alimentos. En este mismo contexto, aparte de perseguir una forma de relación recíproca entre productores y consumidores, también se busca la ruptura con el modelo de explotación de los productores por parte de los intermediarios.

4. En cuanto al contexto espacial y mercantil, se busca reconstruir el imaginario de mercado como un lugar de reencuentro donde se fortalecen las relaciones sociales antes que constituirse como un lugar de simple comercio o de simple circulación de capital económico. Según uno de los entrevistados, este lugar es donde hay encuentros con antiguos amigos, por ello, no es un sitio de negocios, sino que se busca recuperar el verdadero sentido de lo que era el mercado en tiempos anteriores y promover el desarrollo de formas horizontales, solidarias y recíprocas de participación. Por tanto, los consumidores deben involucrarse porque este espacio busca cambiar patrones estructurales de la forma en que se ha llevado la alimentación hasta la actualidad (Fundador CZV, entrevista, 2012).

5. Por último, otro objetivo reforzado en el ámbito de los consumidores, es el de enfatizar en los derechos de los mismos. En este sentido, los consumidores son percibidos como actores que por derecho pueden acceder, exigir y elegir productos sanos y nutritivos, pagar un precio justo y accesible por ellos, y además tienen el derecho de confiar en su producción. Tema que permite cuestionar la función de los sellos verdes y las etiquetas de control alimentario, dado que la cooperativa no cree en este tipo de garantías y ha optado por desarrollar otras alternativas de seguridad como las visitas a las fincas y a los lugares de producción de los alimentos como forma de garantía para los consumidores. Además, organiza talleres y ciclos de cine a fin de concientizar a la población sobre la producción agroecológica y la sustentabilidad. 


\section{La Elvirita y la Red de Guardianes de Semillas}

La Elvirita, al igual que la $\mathrm{CZV}$, es resultado de una cooperativa de productores agroecológicos comprometidos con el consumo responsable. Este mercado inicialmente fue organizado por y es parte de la Red de Guardianes de Semillas (RGS). Dicha red funciona como una "plataforma social dedicada a promover la agrobiodiversidad y la ecología práctica" y se interesa "en construir modelos integrales de vida, trabaja[ndo] en agroecología, bioconstrucción, soberanía alimentaria, economía solidaria, alternativas en educación y tecnologías apropiadas" (Red de Guardianes de Semillas, 2012). De manera que en La Elvirita se encuentran "productos naturales, producidos en casa, de excelente calidad, en esquemas de comercio solidario" (Red de Guardianes de Semillas, 2012). Este colectivo se articula con los circuitos crecientes de ferias agroecológicas y de canastas de barrio, garantizando su calidad a través de la participación de los actores comprometidos con la Red.

La Elvirita, del mismo modo que la CZV, se organiza como una forma de empresa social en la que sus integrantes participan rotativamente en la administración del mercado y las ferias. Según uno de los fundadores de La Elvirita, y a diferencia de la CZV, los productores no están obligados a asistir regularmente a las reuniones administrativas. Sin embargo, todos los participantes del colectivo deben asistir por lo menos a una asamblea anual para tomar decisiones importantes en consenso. La organización de La Elvirita difiere en cuanto a las funciones de los productores, pues en su organización solamente se permite que un solo productor ofrezca determinados artículos, que según la cooperativa sirve para proteger a los productores y evitar la competencia entre productos ofrecidos, a menos que se necesite suplir una demanda elevada o que se dé un acuerdo explícito entre productores (Javier Carrera, entrevista, 2012).

La Elvirita funciona los jueves por la tarde en Tumbaco, y las ferias tienen lugar el segundo domingo de cada mes. En las ferias, el espacio acoge a todos los productores asociados a la Red y los consumidores pueden interactuar directamente con ellos; mientras que en el mercado de La Elvirita, el encuentro será solo con los administradores. Inicialmente, a los consumidores se les proveía de canastas, sin embargo, esto cambió porque muchos consumidores prefieren elegir los productos que van a llevar a sus hogares.

La RGS busca un cambio en el paradigma que gobierna la vida de las sociedades como la ecuatoriana. A partir de esta noción se ha tejido una red que engloba a diversos actores, no solo productores campesinos y urbanos, sino también artesanos y portadores de conocimientos locales, los cuales son revalorizados mediante encuentros, ferias e incluso a través de la impartición de dichos conocimientos a personas interesadas mediante cursos educativos (Red de Guardianes de Semillas, 2012).

El objetivo fundamental de la RGS es la comercialización por parte de los productores hacia los consumidores en términos de justicia social y ambiental. Para mantener este enfoque, han elaborado un sistema de gestión y certificación propio tomado desde la permacultura, en el que cada aspecto cumplido le va dotando de una huella social y ecológica menor, y le otorga mayor calidad agroecológica al producto. De esta manera, es posible rastrear el origen y las externalidades de cada producto, y el consumidor tiene la información adecuada para decidir qué alimentos adquirir (Javier Carrera, entrevista, 2012).

\section{Problemas y limitaciones}

Durante el tiempo que han trabajado juntos, las cooperativas han logrado consolidar al grupo de productores y consumidores, permitiéndoles gastar poca energía y tiempo en la divulgación de sus propuestas para mantenerlas a flote. No obstante, al igual que todo proyecto, estas propuestas han presentado algunos problemas y limitantes en los ámbitos de producción y consumo. Por ejemplo, en el proceso inicial de dichas cooperativas existieron actores que se vincularon desconociendo los objetivos e ideología de las cooperativas, de manera que ocultaron 
la procedencia de sus productos para lucrarse con los alimentos que ofrecían. En otras palabras, participaron personas que afirmaban tener productos orgánicos, pero que en realidad habían sido producidos con agroquímicos y en monocultivos, y que vieron la participación en las cooperativas como una oportunidad de vender sus alimentos a un precio por encima de su valor real.

También se infiltraron intermediarios que habían afirmado ser productores directos. Como solución a este tipo de contrariedades, ambos colectivos de comercialización tienen formas de comprobar y garantizar la continuidad de su propuesta, ya que realizan reuniones con frecuencia, visitan las fincas, los productores se conocen entre sí, se encuentran en otras ferias y son proveedores de otros lugares que requieren alimentos agroecológicos (como la tienda Camari y el restaurante Cassollette).

Por otro lado, a nivel interno lo ideal es que cada productor ofrezca alimentos distintos, sin embargo, todavía hay algunos productores que tienen varios alimentos similares, lo cual genera cierta competencia y rivalidad. Asimismo, existen productores que ofrecen alimentos que tienen mayor demanda que otros, lo que produce -según los niveles de producción y la cantidad de productos ofertados- desigualdad entre productores. Otra limitante consiste en que los productores han tenido que responder al servicio de rentas internas con el pago anual de un impuesto simplificado (RISE) por ser una actividad menor. No obstante, la comercialización de los productos se desarrolla una vez a la semana, lo que implica mayores gastos para una actividad poco lucrativa.

En cuanto a actividades relacionadas con instituciones gubernamentales y no gubernamentales, las cooperativas han tratado de mantener su autonomía. De manera que han decidido no participar en procesos organizativos ni productivos por parte de gobiernos locales ni de organizaciones no gubernamentales. Esto se debe a que según sus experiencias (de participación o de diálogo con otros actores de ferias agroecológicas), el trabajo con este tipo de organizaciones no ha permitido consolidar un grupo estable y, por lo tanto, en vez de fortalecer las redes, las destinan a desaparecer por las dependencias y condiciones que estas imponen (cooperación, normativas institucionales, controles externos, sellos, etc.). Mientras que si actúan con la mayor autonomía posible, estas actividades retoman la importancia real de sus actores, que radica en las actividades productivas como medio para mantener a sus familias, dado que no se trabaja por un sueldo sino para vivir.

Con respecto a los consumidores, algunas limitantes son la distancia, los precios de los productos o el hecho de tener que adaptarse a los horarios de comercialización que se establecen en las cooperativas, ya que los consumidores no necesariamente tienen el tiempo, los medios para adquirir los productos o buscan lugares donde se oferta permanentemente alimentos que no necesariamente son orgánicos ni agroecológicos (como supermercados, micromercados, entre otros).

\section{A manera de conclusión}

Mediante un régimen de soberanía alimentaria se apuesta por un modelo donde los actores se orientan primordialmente hacia la producción de cultivos para el autoconsumo, la venta directa y la compra de productos necesarios a nivel local, a la vez que se incentiva la generación local de empleo. Estas condiciones no se restringen al ámbito rural, sino que sus efectos también se extienden a los sectores urbanos, donde las ferias agroecológicas y los mercados alternativos desempeñan un papel importante en la reducción y eliminación de las fronteras existentes entre el campo y la ciudad, motivando a la vez la relación directa entre agricultores y consumidores. En este sentido, se busca que los productores vendan sus alimentos a precios justos y sin intermediarios, mientras que los consumidores compren alimentos variados, culturales y nutritivos, conociendo sobre su procedencia y forma de cultivo. Esta estrategia se orienta hacia un flujo de ganancias que circula al interior de las ferias, cooperativas o colectivos, enfrentando 
la lógica del modelo económico dominante criticado por Rosset, quien argumenta que cuando las producciones campesinas se exportan a bajos precios impuestos por el mercado internacional y gran parte de los productos de consumo se importan a altos precios, el flujo de ganancias sale de la economía local hacia las economías externas (Rosset, 2004: 2). En efecto, la consolidación de la percepción de soberanía alimentaria y sus múltiples componentes, incluidos la agroecología y los circuitos de producción y consumo local, deben ser piezas elementales para el impulso del desarrollo económico.

Por otro lado, los modelos alternativos de producción y consumo expuestos dependen de su interacción con diferentes actores, ya sean los relacionados con la producción, la distribución o el consumo. Incluso dentro de cada uno de estos ámbitos, se encuentra una diversidad de dinámicas como las resistencias de los pequeños productores frente a la producción de mediana y gran escala, además de elementos de carácter de género e identidad. Sin embargo, el objetivo es común, los colectivos agroecológicos buscan reconfigurar la organización de la producción y circulación de los alimentos, bajo un modelo que difiera de la dinámica tradicional de acumulación capitalista. A pesar de ello, no se puede asegurar que estas iniciativas verdaderamente constituyen un contrapeso al régimen corporativo, pues si bien se enfocan en una mejoría de las prácticas productivas, aún existen grandes desigualdades en el control de la producción y el comercio.

La generación de nuevas iniciativas agroecológicas en los barrios y vecindarios es esencial para la transformación social y ecológica. En este artículo se mencionó que las canastas comunitarias surgieron como una necesidad para satisfacer la adquisición de alimentos para un sector de la población con insuficientes recursos económicos, sin embargo, las iniciativas estudiadas se orientan hacia consumidores que no necesariamente tienen estas mismas características, lo cual indica un creciente interés por consumir alimentos agroecológicos u orgánicos. Este creciente interés de los consumidores apoya directamente a los pequeños productores, pues las técnicas que se utilizan en la agroecología se reproducen en este sector. En este sentido, se constató que los consumidores constituyen un motor de cambio, porque orientan la producción según lo que deciden comprar.

Por otro lado, esta visión tiene sus límites, pues si la oferta se centra mayoritariamente en productos que no son necesariamente agroecológicos, pero promueven beneficios en la salud por ser producidos sin agroquímicos, como el caso de los alimentos conocidos como orgánicos, estaríamos enfrentando un fenómeno de medicalización de la alimentación. Esta situación se presenta como una desventaja para el sector productivo, porque deja de lado la reflexión sobre las condiciones de producción y la sobreexplotación de los campesinos y el medioambiente, a la vez que podría estar reproduciendo cadenas de valor agrícolas determinadas por las corporaciones o los agronegocios a gran escala. En este contexto, los consumidores optan por buscar alimentos sin considerar las condiciones de trabajo de los productores, únicamente el componente de la salud. Por lo tanto, las grandes empresas y los productores se orientarán a ofertar este tipo de productos, alimentos que incluso hoy en día encontramos en crecientes proporciones y con precios relativamente más altos que los productos convencionales en los supermercados.

Dada esta observación, el reto podría quedar en la mediación de precios que proponen los colectivos y el mercado. Si los productores de las iniciativas agroecológicas sostienen costos asequibles para la población, los productos podrán distribuirse favorablemente, pero si los costos son más elevados que los ofertados en los mercados tenderán a competir, repitiendo la misma estructura de distribución y comercio alimentario que ha permanecido en los dos últimos regímenes alimentarios indicados por McMichael. Sin embargo, creemos que el tratamiento de la producción de alimentos agroalimentarios más que en los costos, debe ser de carácter estructural, donde es imperativo que el Estado regule la distribución de la riqueza y los recursos de la naturaleza, y la sociedad civil proteja, promueva y reproduzca lógicas distintas a las de un sistema económico mercantil, como las que se han llevado a cabo a través de la solidaridad, la reciprocidad, el trueque, etc., dentro de las cooperativas. 
Finalmente, por diversas razones señaladas en este texto, el papel desempeñado por el Estado en cuanto a la protección de la soberanía alimentaria muestra una interacción dialéctica bastante frágil en la relación entre la teoría y la realidad. Al conectar el régimen agroindustrial mundial con las políticas públicas desempeñadas en el ámbito nacional, nos encontramos con argumentos directos o indirectos que se dirigen a favor de la regulación, planificación e intervención del sector público, que reproducen el modelo del tercer régimen alimentario de McMichael y dentro de él, la segunda contradicción del capitalismo propuesta por $\mathrm{O}^{\prime}$ Connor, donde la naturaleza se sigue reproduciendo como una mercancía. 


\section{Bibliografía}

Altieri, Miguel y Nicholls, Clara. 2010, “Agroecología: potenciando la agricultura campesina para revertir el hambre y la inseguridad alimentaria en el mundo", en: Revista de Economía Crítica No 10, pp. 62-74.

Altieri, Miguel y Toledo, Víctor. 2011, "The agroecological revolution of Latin América: rescuing nature, securing food sovereignity and empowering peasants", en: Journal of peasant Studies 38, 3, pp. 587-612.

Andrade, Diego y Flores, Miguel. 2008, El consumo de productos orgánicos/agroecológicos en los hogares ecuatorianos, VECO Ecuador, Quito.

Arce, Alberto y Marsden, Terry. 1993, "The social construction of international food: a new research agenda", en: Economic Geography 69, 3, pp. 293-310.

Borja Hidalgo, Patricia. 2009, "Las directrices sobre derecho a la alimentación y su correspondencia con la Constitución del Ecuador", Afese No. 52, pp. 103-120.

Bretón, Víctor. 2009, “Continuarán muriendo de hambre millones de personas en el siglo XXI?”, en: Revista Española de Estudios Agrosociales y Pesqueros No. 224: pp. 69-109.

Carrión, Diego y Herrera, Stalin. 2012, Ecuador rural del siglo XXI, Instituto de Estudios Ecuatorianos, La Tierra, Quito.

Constitución Política de la República del Ecuador. 2008.

Cooperativa Zapallo Verde. 2008, ¿Por qué ser parte de la cooperativa zapallo verde?, Cooperativa Zapallo Verde, visita 26 de diciembre de 2012 en:http://zapalloverde.blogspot.com/2008/02/por-qu-ser-parte-de-la-cooperativa.html

COPISA. 2011, Lineamientos para una propuesta de agrobiodiversidad, semillas y agroecología, visitado en agosto 2, 2012; disponible en: http://www.soberaniaalimentaria.gob.ec/pacha/wp-content/uploads/ 2011/04/ Lineamientos-para-una-Ley-de-Agrobiodiversidad.pdf.

FAO. 2012, Las cooperativas son cruciales en la lucha contra el hambre: Porto Alegre/Roma, FAO, visita 28 de diciembre de 2012 en http://www.fao.org/news/story/es/item/120796/icode/

Garcés, Sandra y Kirwan, Emma. 2009, "Las Canastas comunitarias en Ecuador: una apuesta por la salud, la economía y la solidaridad", en: Revista Letras Verdes $\mathrm{N}^{\circ} .5$, pp. 9-11.

Idrovo, Jorge. 2016, “Transformaciones rurales y agrarias en Ecuador”, en: Serie Documento de Trabajo No. 179, RIMISP, Santiago de Chile.

Kirwan, Emma. 2008, "La canasta comunitaria: una plataforma urbano-rural para la seguridad alimentaria" en: LEISA Revista de Agroecología $\mathrm{N}^{\circ} 24$ pp. 26-29.

Larach, María Angélica. 2001, El comercio de los productos transgénicos: el estado del debate internacional, CEPAL, Santiago de Chile.

Long, Norman. 2007, Sociología del desarrollo: una perspectiva centrada en el actor, Ciesas, México D.F.

LORSA, Ley Orgánica del Régimen de la Soberanía Alimentaria. 2010.

McMichael, Philip. 2015, Food regimes and agrarian questions, Fernwood Press, Halifax.

Montagut, Xavier. 2011, “Introducción”, en: Jordi Gascon y Xavier Montagut (Coord.) Estado, movimientos sociales y soberanía alimentaria en América Latina: ¿Hacia un cambio de paradigma agrario?, Flacso, Quito.

Novoa, Braulio. 2013, “Soberanía alimentaria, consumo, acceso a alimentos y nutrición”, en: Hidalgo, Francisco, Pierril Lacroix y Paola Román (Eds.) Comercializacion y Soberanía Alimentaria, SIPAE, Quito, pp: $115-131$.

O`Connor, James. 2001, Causas naturales: ensayos de marxismo ecológico, Siglo XXI editores, México.

ONU. 2011, 2012 año internacional de las cooperativas, ONU, visita 28 de diciembre de 2012 en http://www. un.org/es/events/coopsyear/

Perfecto, Ivette et al. 2009, Nature's Matrix: Linking Agriculture, Conservation and Food Sovereignty, Earthscan, London.

Ploeg, Jan Douwe van der. 2010, Nuevos campesinos: campesinos e imperios alimentarios, Icaria, Barcelona.

Red de Guardianes de Semillas. 2007, “Qué es la Soberanía Alimentaria?”, en: Allpa Boletín de agroecología y semillas, $\mathrm{N}^{\circ} 6$, pp. 1-5.

Red de Guardianes de Semillas. 2012, Red de Guardianes de Semillas, visitado el 26 de diciembre de 2012 , disponible en: http://www.redsemillas.org/.

Rosero, Fernando et al. 2011, Soberanía alimentaria, modelos de desarrollo y tierras en Ecuador, CAFOLIS, Quito.

Rosset, Peter. 2009, "Food Sovereignity in Latin America: Confronting the New Crisis", en: NACLA Report on the Americas, May-June, pp. 16-21.

2004, "Soberanía Alimentaria: Reclamo Mundial del Movimiento Campesino", en: Adriana Latrónico y María Elena Martínez (Trads.), Food Sovereignty: Global Rallying Cry of Farmer Movements, Institute for Food and Development Policy Backgrounder Vol. 9, N 4, Fall 2003, pp. 1-4.

WWF. 2008, Informe planeta vivo 2008, World Wildlife Fund, Cali. 